\title{
Calculation Algorithm for Diffraction Losses of Multiple Obstacles Based on Epstein-Peterson Approach
}

\author{
Ahmad S. Abdulrasool, ${ }^{1}$ Jabir S. Aziz, ${ }^{2}$ and Sadiq J. Abou-Loukh ${ }^{1}$ \\ ${ }^{1}$ Electrical Engineering Department, Baghdad University, Baghdad, Iraq \\ ${ }^{2}$ Electronics and Communication Engineering Department, Al-Nahrain University, Baghdad, Iraq \\ Correspondence should be addressed to Ahmad S. Abdulrasool; ahmadsalam5101986@gmail.com
}

Received 25 April 2017; Revised 2 July 2017; Accepted 27 July 2017; Published 3 October 2017

Academic Editor: Huapeng Zhao

Copyright @ 2017 Ahmad S. Abdulrasool et al. This is an open access article distributed under the Creative Commons Attribution License, which permits unrestricted use, distribution, and reproduction in any medium, provided the original work is properly cited.

Applying propagation models with good accuracy is an essential issue for increasing the capacity and improving the coverage of cellular communication systems. This work presents an algorithm to calculate total diffraction losses for multiple obstacles objects using Epstein-Peterson approach. The proposed algorithmic procedure to model the diffracting can be integrated with other propagation mechanisms in ray-tracing for the prediction of received signal level in non-line-of-sight environments. This algorithm can be interpreted into software application to scan large areas with a reasonable simulation time.

\section{Introduction}

With the rapid development of networks and wireless communication, there is an increasing need for reliable point-toarea prediction tools in the planning of radio communication services. These prediction tools must include the characteristics of the particular zone in which the system is intended to be used [1]. The wireless network sites/cells/access points are distributed based on the traffic and path loss and usually it appears like the one shown in Figure 1.

Propagation mechanisms may generally be attributed to reflection, diffraction, and scattering. Diffraction and scattering occur when the obstructing object is large compared to the wavelength of the radio wave. Diffraction allows radio signals to propagate around the curved surface of the earth, beyond the horizon, and behind obstructions. A complex environment with obstacles may attenuate the electromagnetic waves. If the obstacle will diffract the wave over or around the obstruction, there will be high attenuation through the obstacle [2]. Determination of the diffraction losses with a minimum acceptable error is very challenging for realistic propagation environments such as mountainous regions, so that system design requires a set of statistics that describes the expected performance of a propagation path. Propagation models are employed to provide the required statistics. A propagation model can be a set of mathematical expressions, diagrams, and/or algorithms used to represent the radio characteristics for a given environment. The pathloss prediction models can be roughly divided into three types, empirical, theoretical, and site-specific [3].

An empirical model is usually a set of equations derived from extensive field measurements. The main advantage of empirical models is their simplicity and computational efficiency. The model accuracy depends on the measurement accuracy and the similarity between the environments where the measurements and predictions are taking place [4]. The input parameters for the empirical models are usually qualitative and not very specific, for example, a dense urban area and a rural area. One of the main drawbacks of empirical models is that they cannot be used for different environments without modification [5].

A theoretical model is based on the principals of physics and may therefore be applied to different environments without affecting the output. The main drawbacks of theoretical models are the wide range error, which is impractical to base networks design and optimization on such models.

A site-specific model is based on numerical methods such as the ray-tracing method and the finite-difference time-domain (FDTD) method. The input parameters can be very detailed. Ray-tracing is a method that uses a geometric 


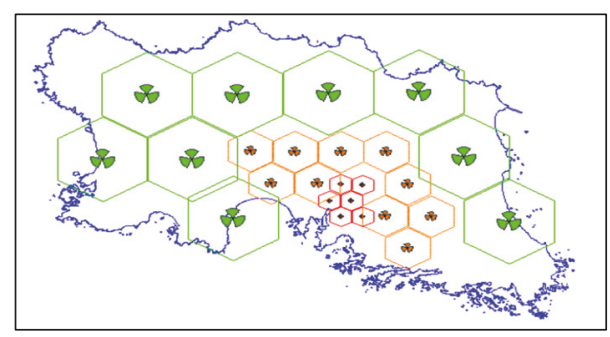

Figure 1: Example of cellular network plan.

approach and examines what paths the wireless radio signal takes from transmitter to receiver as if each path was a ray of light (possibly reflecting from the surfaces) as shown in Figure 2 [6]. Ray-tracing predictions are good when detailed information of the area is available, but the predicted results may not be applicable to other locations, thus making these models site-specific. The disadvantages of site-specific models are the large computational overhead that may be prohibitive for some complex environments as stated in $[7,8]$.

The ray-tracing accuracy and overhead computation is largely dependent on the method of diffraction loss calculation. There are many methods for diffraction calculation purpose; each differs in complexity and accuracy. The most used methods are the Bullington [9], Deygout [10], and Epstein-Peterson [11]. These methods are not presented in algorithmic form that is suitable for software integration; thus it is hard to find diffraction loss for large areas using these methods.

This work focuses on the diffraction loss calculation for multiple obstacles and presents an algorithm for the process of calculation that can be interpreted into software application for coverage prediction tools.

\section{Diffraction}

Diffraction is the propagation of wave behind obstacle even when the line-of-sight (LOS) is not clear (non-line-of-sight (NLOS)) as shown in Figure 3. Due to diffraction, coverage still presented even when the RF signal is obstructed. The physical and the mathematical explanation of this phenomenon are detailed in [12].

In order to get good result for prediction, the RF diffraction should be taken into consideration. Since coverage will extend behind obstacles (cell size increased), signal level estimation will increase at many points in certain geographical areas. Also the quality of signal at the mobile station will be impacted if it is getting power from cells other than the serving cell (due to coverage extension of the other cells due to diffraction). Capacity will be impacted as well due to this coverage extension as it will serve a larger geographical area and hence more mobile stations under the same serving cell.

The diffraction is dealt with as a kind of loss, usually measured in $\mathrm{dB}$. This amount of loss is directly subtracted from total signal power.
Diffraction loss (DL) is calculated as follows by approximating the shape of obstruction object to knife edge [13]:

$$
v=h \sqrt{\frac{2\left(d_{1}+d_{2}\right)}{\lambda d_{1} d_{2}}} .
$$

If $v \leq-1$

$$
\mathrm{DL}=0 .
$$

If $-1<v \leq 0$

$$
\mathrm{DL}=20 * \log _{10}(0.5-0.62 * v) .
$$

If $0<v \leq 1$

$$
\mathrm{DL}=20 * \log _{10}\left(0.5 * e^{-0.95 * v}\right)
$$

If $1<v \leq 2.4$

$$
\begin{aligned}
\mathrm{DL}= & 20 \\
& * \log _{10}\left(0.4-\sqrt{0.1184-(-0.1 * v+0.38)^{2}}\right) .
\end{aligned}
$$

If $2.4<v$

$$
\mathrm{DL}=20 * \log _{10}\left(\frac{0.225}{v}\right)
$$

$v$ is the diffraction parameter. $h$ is the obstacle height in meters. $d_{1}$ is the distance between cell and obstacle in meters. $d_{2}$ is the distance between mobile and obstacle in meters. $\lambda$ is the signal wavelength in meters.

These parameters are medicated in Figure 3.

\section{Multiple Obstacles Diffraction}

Usually, when the signal propagates from transmitter to receiver it exhibits from zero to $n$ multiple obstacles. Each obstacle will cause diffraction and the received signal level is governed by the net diffracting loses plus other propagation mechanisms effects. Calculation of multiple objects diffraction losses is subjected to many models under the Uniform Theory of Diffraction (UTD). Each model differs in complexity and accuracy that varies from one propagation environment to another. The most famous models are Bullington, Deygout, and Epstein-Peterson.

The Epstein-Peterson [11] is a diffraction model that considers all the obstruction objects. It produces acceptable error, but the error increases when the obstruction objects are closely spaced. Even though Epstein-Peterson model produces less error than Bullington model, it is systematic compared to Deygout model as stated in $[3,14]$.

The Epstein-Peterson model regards the first obstruction object as new signal source. The next step is to consider the next obstruction object (between the new source and the receiver) as the new source. Every time move one step to the next obstruction object and redo these steps till there is no more obstruction object left. The net diffraction loss is the sum of diffraction lost per each step. 


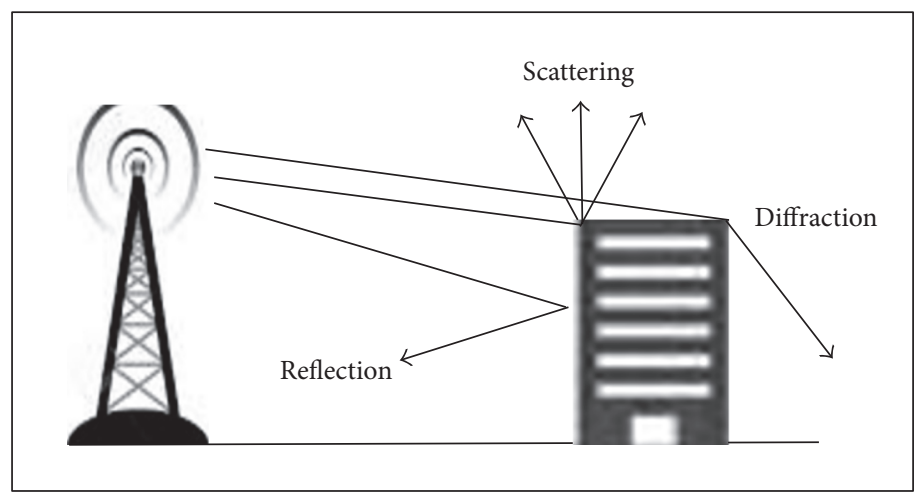

FIGURE 2: Propagation mechanisms in ray-tracing.

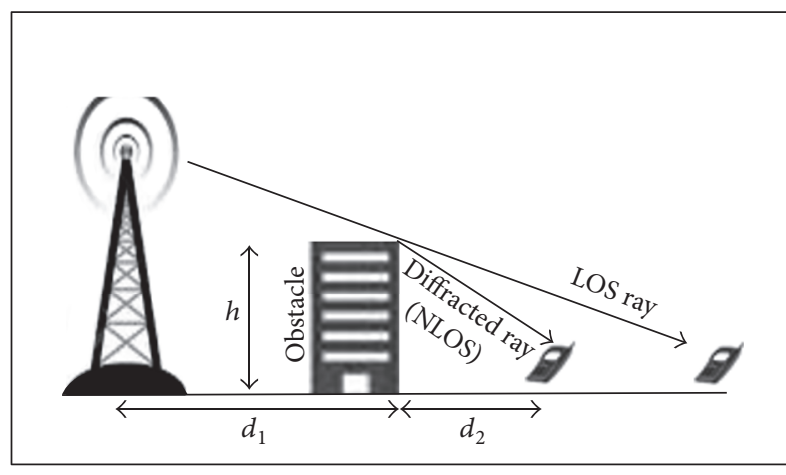

Figure 3: Diffraction of rays when line-of-sight is obstructed.

\section{Multiple Obstacles Diffraction Losses Calculation Algorithm}

As stated in the previous section, the Epstein-Peterson approach is systematic with acceptable error (unless obstruction obstacles are closely spaced). In this work, an algorithm for calculating the total diffraction losses based on Epstein-Peterson approach for a defined geographical area is proposed. The steps of the algorithm are shown in Figure 4.

The following is the summary of the algorithm's variables in the flow chart with definitions.

DB is the cellular network data base.

cell $h$ is the specified cell height (antenna height above the ground plus the elevation on $3 \mathrm{D}$ map) (in meters).

$I$ and $J$ are the geographical square dimensions (unitless integers).

ms $h$ is an $I$ by $J$ matrix which represents the mobile height at $3 \mathrm{D}$ map (average mobile height plus the elevation on 3D map) (in meters).

$i, j, k$, and $m$ are counters.

$\mathrm{ms}(i, j)$ is the mobile located in $i$ th and $j$ th point on 3D map.

$\operatorname{PP}(i, j)$ is the path profile between cell and $\operatorname{ms}(i, j)$ (unitless).
$K$ is number of objects (hills, building, houses, trees, and etc.) in $\operatorname{PP}(i, j)$ (unitless integers).

$M$ is the number of obstacles in $\operatorname{PP}(i, j)$.

$h_{\mathrm{ec}}$ is the increase in object height due to earth curvature (in meter).

$\operatorname{Obj} \operatorname{loc}(k)$ is the $k$ th object location in $\operatorname{PP}(i, j)$ (in meters).

Obj $h$ is the $k$ th object height in $\operatorname{PP}(i, j)$ (in meters).

$A, B, C, D, E$, and $F$ are temporary variables.

Obst_loc $(m)$ is the $m$ th obstacle location in $\operatorname{PP}(i, j)$ (in meters).

Obst_h(m) is the $m$ th obstacle height in $\operatorname{PP}(i, j)$ (in meters).

Obst_DL $(m)$ is the diffraction losses resulting from the $m$ th obstacle (in $\mathrm{dB}$ ).

ms_DL $(i, j)$ is the total diffraction losses experienced by $\mathrm{ms}(i, j)($ in $\mathrm{dB})$.

The following are the footnotes in the flowchart:

(1) Load cellular network data base (DB).

(2) Load the 3D map zone over which the processing will be implemented. The 3D maps are segmented in order to reduce amount of computations by excluding unnecessary areas.

(3) Get geographic square from 3D Map with dimensions equal to max technology coverage multiplied by 2 and have center at the same cell location. This setup will reduce the computation further by excluding the areas which cannot be served by a cell due to technology limitation. For example, GSM technology has max cell radius of $32 \mathrm{Km}$; thus it is not required to calculate the diffraction loss outside this radius.

(4) Set $I$ and $J$ to be equal to the dimensions of the geographic square dimensions obtained from the previous step.

(5) Extract path profile $(\mathrm{PP}(i, j))$ between cell and $\mathrm{ms}(i, j)$ from the 3D map.

(6) Mountains, hills, buildings, tress, and others are objects that may or may not obstruct the LOS.

(7) Object location and object dimension are found from $\mathrm{PP}(i, j)$ stored in $\mathrm{Obj} \operatorname{loc}(k)$ and $\mathrm{Obj} h(k)$, respectively. 

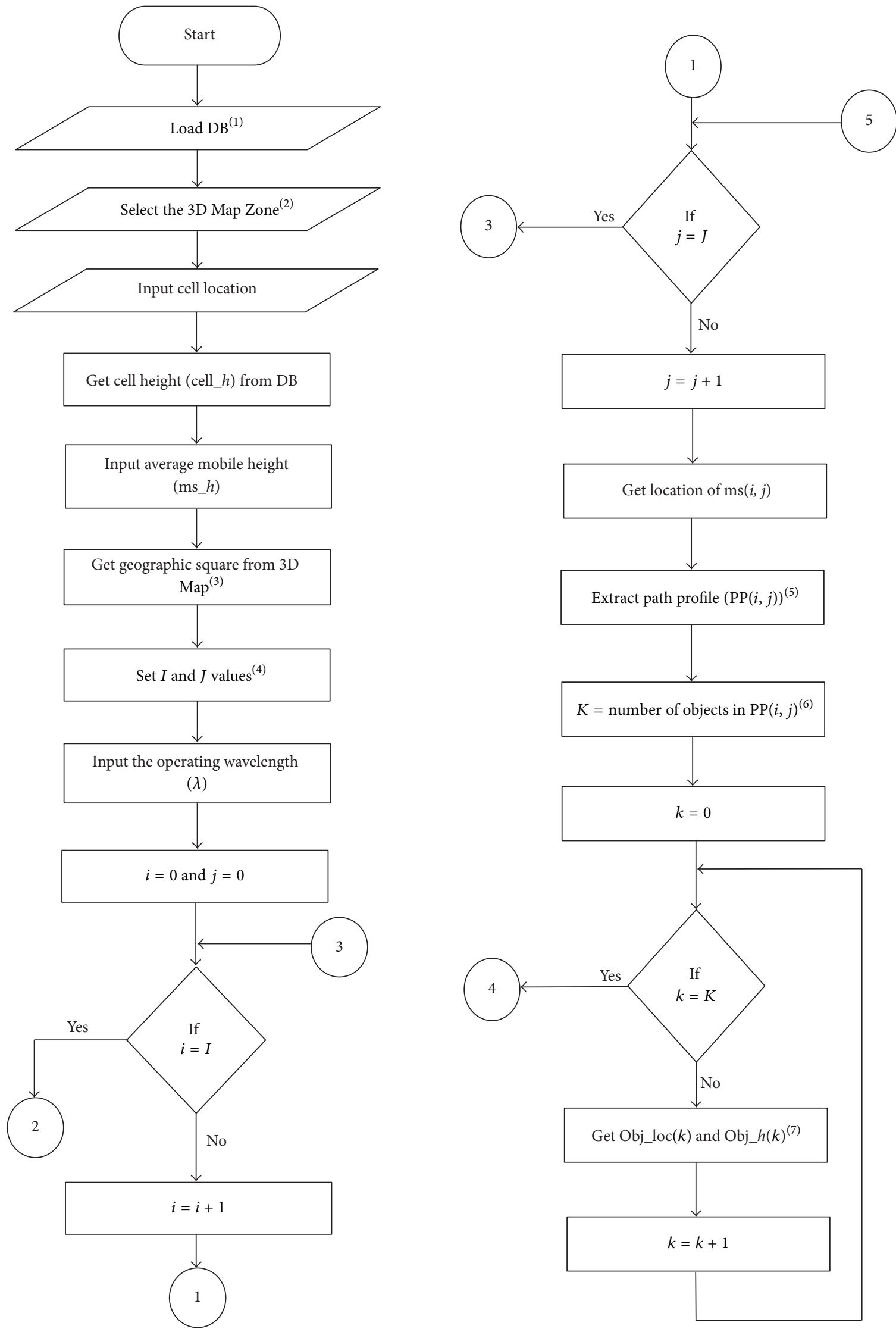

FIgure 4: Continued. 


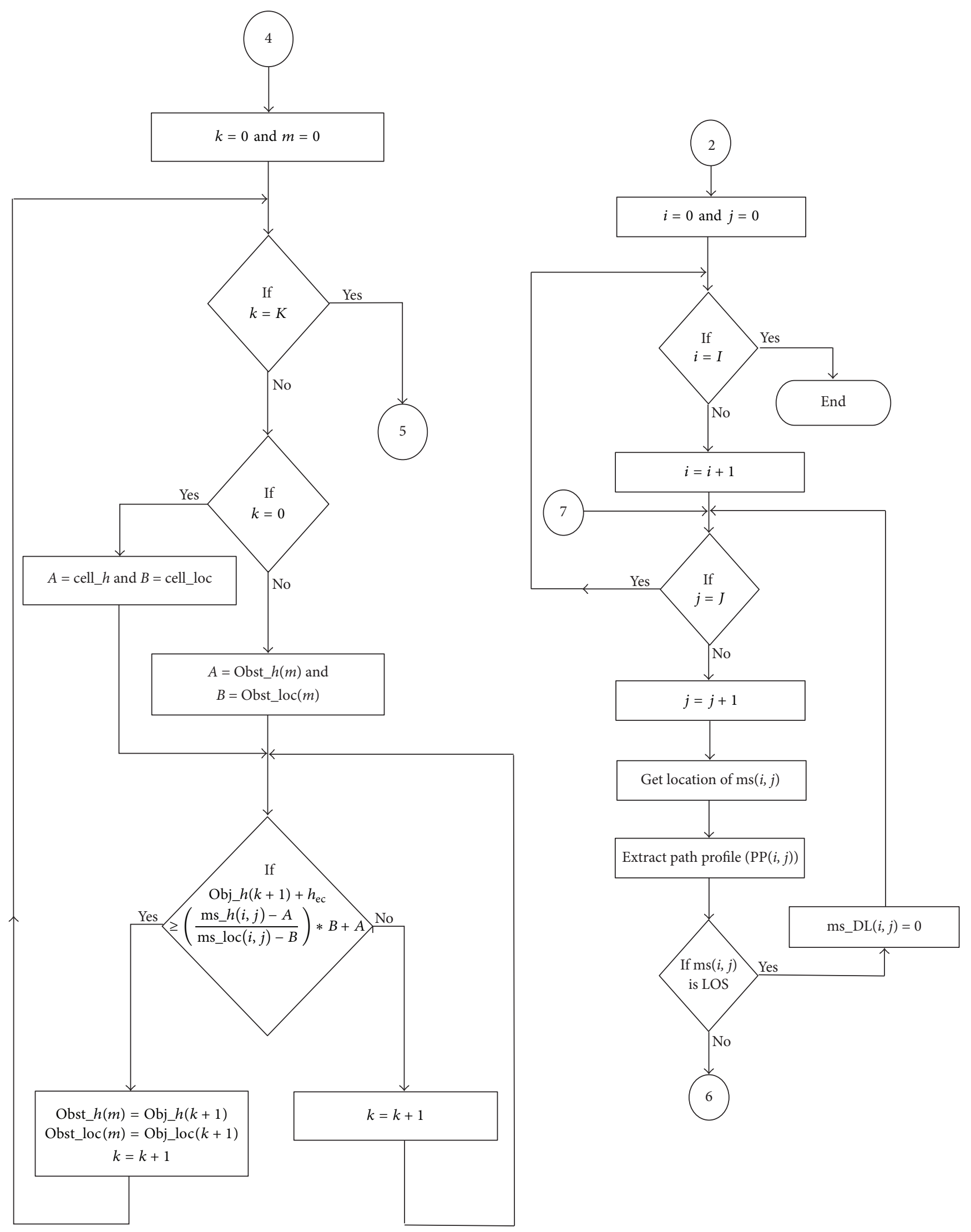

FIgURE 4: Continued. 


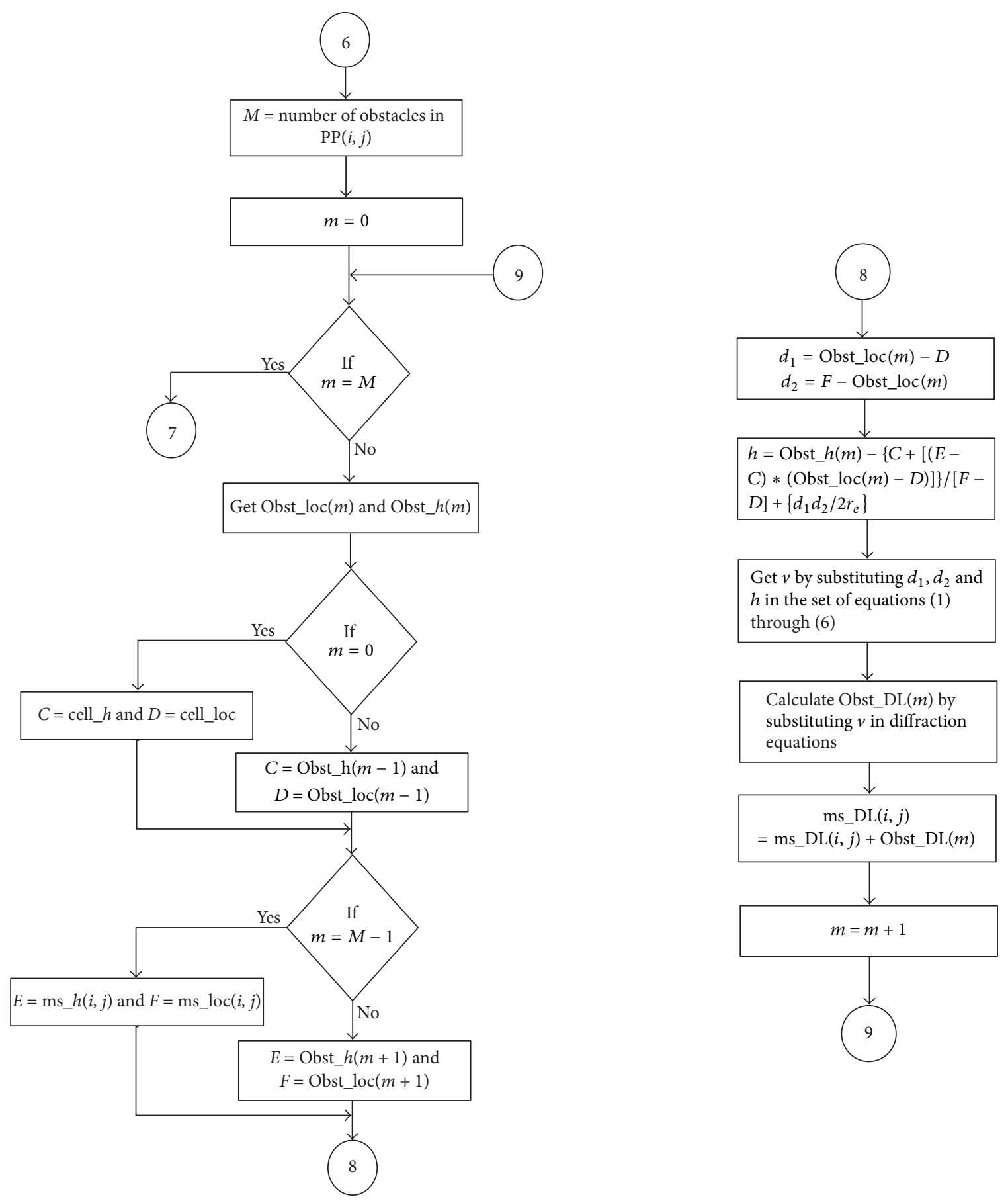

FIGURE 4: Multiple obstacles diffraction losses calculation algorithm flow chart.

\section{Practical Implementation of Methodology}

In order to validate the proposed diffraction loss calculation methodology, a comparison needs to be made between simulation and practical measurement. It is well known that the diffraction of RF signal cannot be measured directly since it is combined with other propagation mechanisms that contribute to RF signal level through the interaction with propagation environment's elements.
In this work, all the propagation mechanisms (including the diffraction loss) will be simulated under ray-tracing propagation model. The simulated signal level resulting from this model will be used for the comparison with the measured signal level using Root Mean Square Error (RMSE). This comparison will be used to validate the proposed methodology. The RMSE will give an indication of the diffraction mechanism simulation accuracy if other propagation mechanisms are simulated with minimum possible error. 


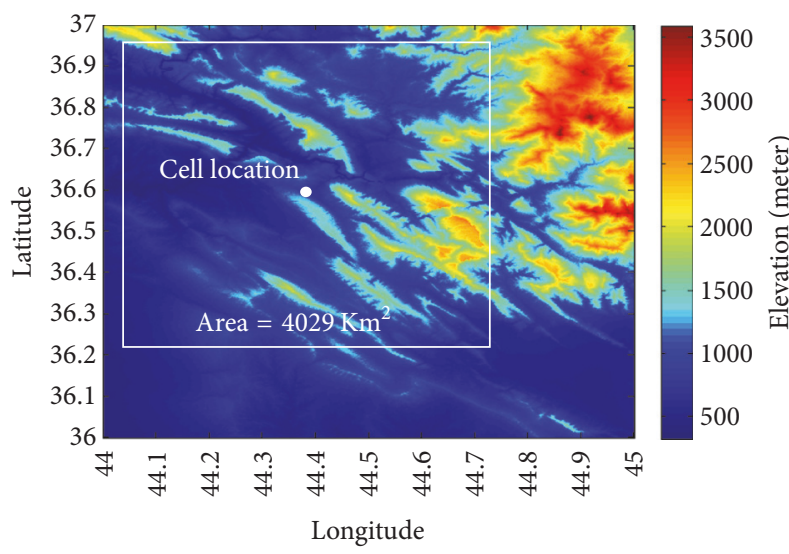

FIGURE 5: Map for the methodology test.

The ray-tracing model was used to simulate signal propagation from GSM cell using the following data:

(i) Geographical zone is between latitude $36^{\circ}-37^{\circ}$ and longitude $44^{\circ}-45^{\circ}$ with an area of $10019 \mathrm{Km}^{2}$. This zone is mountains shown in Figure 5.

(ii) The foliage density is low.

(iii) GSM cell is located at latitude $36.6001^{\circ}$ and longitude $44.3973^{\circ}$.

(iv) Operating frequency is within $900 \mathrm{Mhz}$ band $(0.33 \mathrm{~m}$ wavelength).

(v) Mobil station sensitivity is $-102 \mathrm{dBm}$ (GSM standard).

(vi) The transmitted pilot signal power is $15 \mathrm{~W}(41.7 \mathrm{dBm})$.

(vii) The used antenna model is Tongyu 182018DE-65P with max gain of $17.8 \mathrm{dBi}$ and horizontal beamwidth of $63^{\circ}$ and vertical beamwidth of $7.5^{\circ}$. The provided radiation pattern data is $1^{\circ}$ resolution.

(viii) The antenna physical parameters are $0^{\circ}$ mechanical tilt, $1^{\circ}$ electrical, and $40^{\circ}$ azimuth.

(ix) Cell antenna height is $39 \mathrm{~m}$ height above ground.

(x) The average mobile station height is 0.5 meter (assuming that the mobile is in user pocket sitting in car).

(xi) 3D map in DEM format with $90 \mathrm{~m}$ resolution.

(xii) The ray-tracing was simulated using the MATLB software package.

The diffraction loss calculation will be applied on a part of the $10019 \mathrm{Km}^{2}$ area, and the selection is done autonomously by the algorithm based on cell location and the max radius the technology can cover. For example, in GSM, the max cell radius is $32 \mathrm{Km}$; thus the area on which the simulation is applied is $32 * 2$ by $32 * 2 \mathrm{Km}^{2}\left(4029 \mathrm{Km}^{2}\right.$ out of $\left.10019 \mathrm{Km}^{2}\right)$. This will contribute in a great reduction in simulation time and processing as many points on the $3 \mathrm{D}$ map are excluded as operating technology cannot serve.

The signal level measurements were performed on the road intersected with the prediction map. The measurements

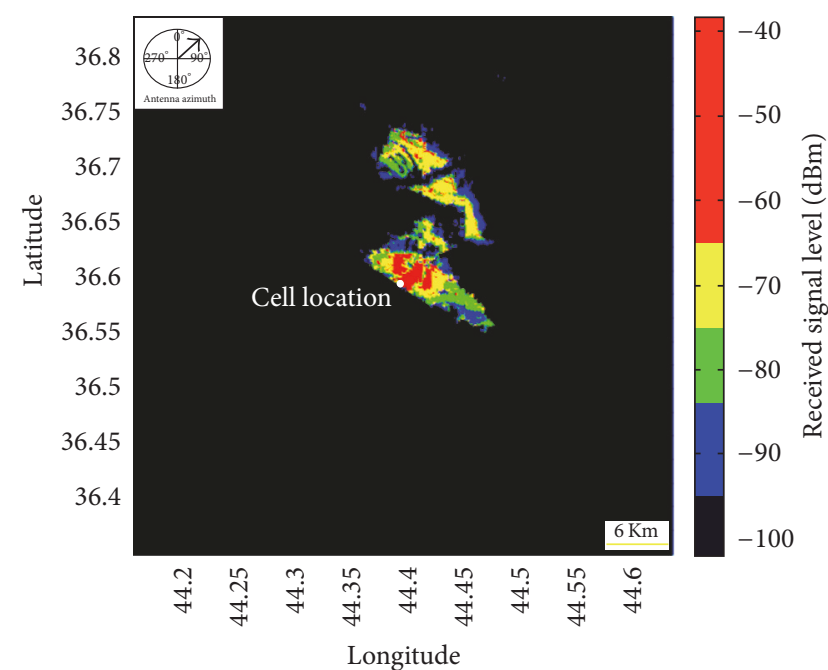

FIGURE 6: Measurement route (pink path) overlaid on prediction map.

were implemented with the mobile station forced-camping on the same cell. Handovers or cell reselections were not allowed; thus the measurement continued on the same cell till there is no more coverage. The resulting measurement route is shown in Figure 6 in the pink route overlaid on the prediction map. The measurement route length is $5.8 \mathrm{Km}$. A GPS device was connected to capture the coordinates at every measurement in order to compare it with the corresponding predicted value.

It should be noted here that the diffraction losses are not required to be calculated every time for cellular networks optimization process. For example, antenna azimuth, tilt, or pilot signal power changes do not imply to calculate the diffraction losses rather than other propagation mechanisms. Also, if a network site has more than one cell (which is usually the case) and these cells' antennas are at the same height, it is enough to do the diffraction calculation for one cell only and assume that it is the same for the other cells.

\section{Results and Discussions}

The diffraction loss map obtained using the proposed algorithm is shown in Figure 7 presented in thematic view; each color represents a certain loss value. The diffraction loss data in Figure 7 is one of propagation mechanisms that are required to obtain the prediction map shown in Figure 6.

In order to examine the result, the measured and predicted received signal level need to be compared. Figure 8 shows the measured and predicted received signal level versus distance. The RMSE of the measured and predicted values versus distance is shown in Figure 9. The overall RMSE is $5.34 \mathrm{~dB}$ with error ranging from $0.0014 \mathrm{~dB}$ to $21.56 \mathrm{~dB}$.

In RF signal propagation, there are two cases for path description for between transmitter and receiver, LOS case and NLOS case. For the NLOS case, the diffraction is the main propagation mechanism that allows RF signal to be intercepted by the receiver. Accordingly, the RMSE will be filtered more. 


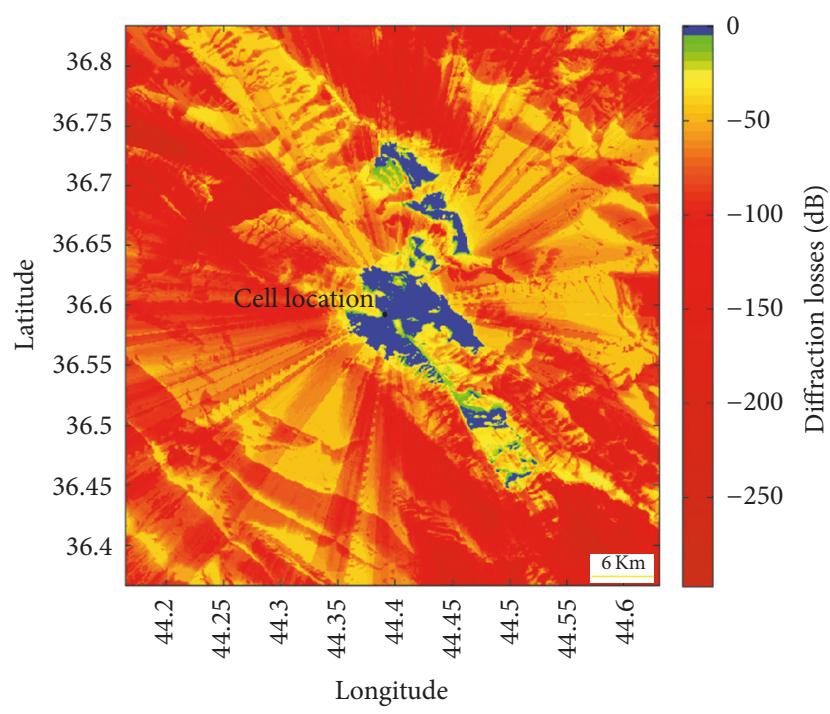

FIGURE 7: Diffraction loss thematic map view $\left(\right.$ area $\left.=4029 \mathrm{Km}^{2}\right)$.

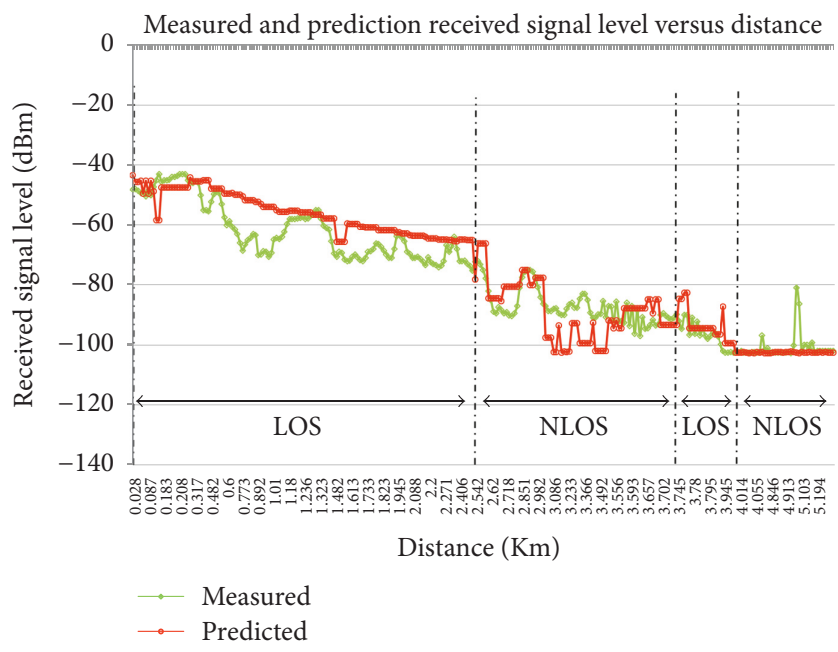

FIGURE 8: Measured and predicted signal level versus distance.

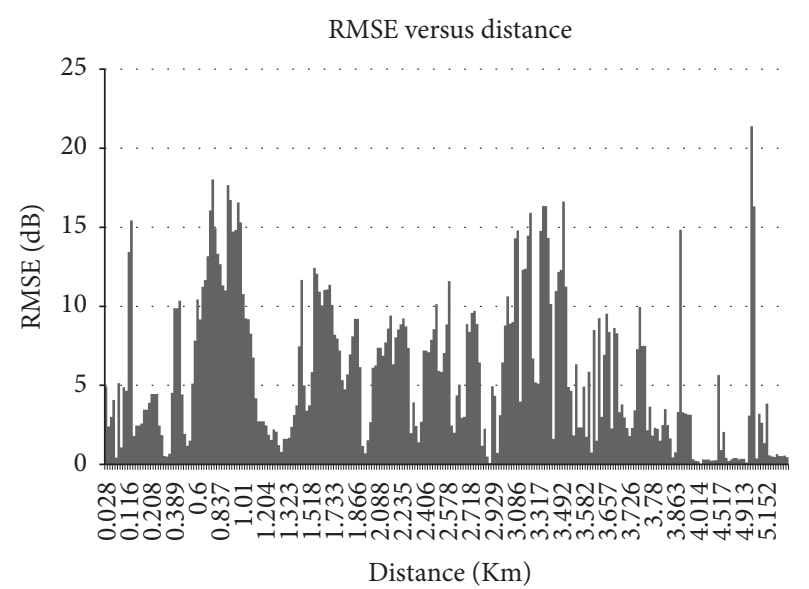

FIGURE 9: Measurement and prediction of RMSE versus distance.

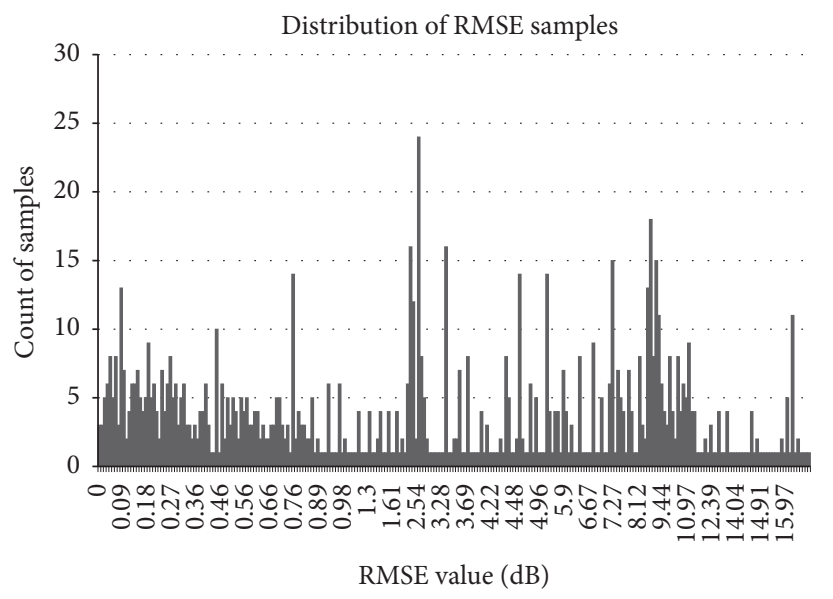

FIGURE 10: RMSE samples distribution for NLOS cases.

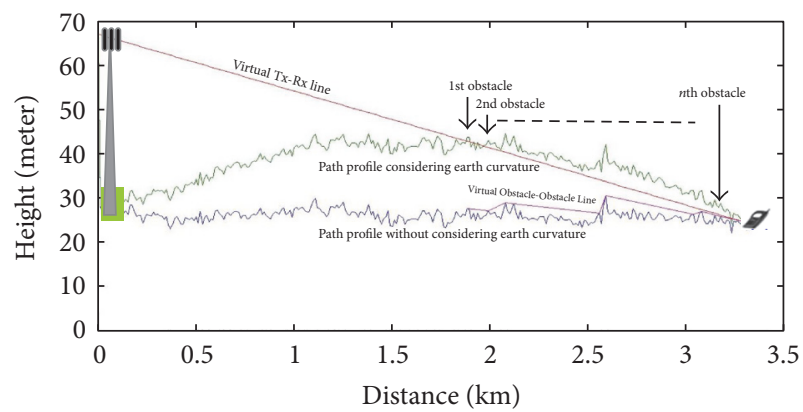

FIgURe 11: Path profile and earth curvature effect.

The RMSE samples distribution is shown in Figure 10. The overall RMSE for NLOS cases is $4.37 \mathrm{~dB}$ with error ranging from $0.0014 \mathrm{~dB}$ to $21.56 \mathrm{~dB}$. Even though the max error is $21.56 \mathrm{~dB}$, the count of these samples is very less.

It can be noted form Figure 7 that the diffraction losses increase as the distance in the radial direction increases. This effect is due to earth curvature and the possibility of increasing the number of obstruction obstacles as shown in Figure 11 .

It was found that same number of obstacles does not give same diffraction loss as shown in Figure 12. This is due to the fact that for a specific number of obstacles there are many possibilities of arrangements (i.e., location of obstacles with respect to each other). The arrangement is parametrized by $h$, $d_{1}$, and $d_{2}$ in (1). This will result in different $v$ values and thus different diffraction loss for the same number of obstacles.

The required simulation time per area of $4029 \mathrm{Km}^{2}$ is 122 minute on PC with core i5 and 8 GB RAM. There is no need to repeat the simulation for a life cellular network optimization purpose.

\section{Conclusions}

By considering the NLOS cases only, the signal propagation modeling using ray-tracing resulted in RMSE of $4.37 \mathrm{~dB}$. This RMSE is attributed to proposed diffraction loss calculation 


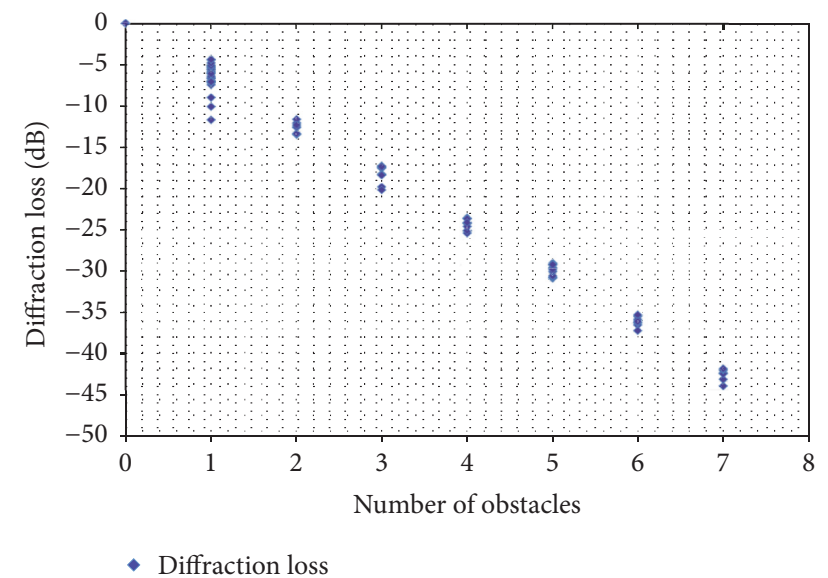

Figure 12: Diffraction losses versus number of obstacles.

algorithm and other propagation mechanisms calculation. For RF signal propagation prediction, this error value is acceptable. Thus it can be concluded that the performance of proposed procedure is acceptable.

The structure and systematic property of the algorithm make it very appropriate for software integration. Due to the acceptable error value and the ability of algorithm to cover very wide areas, the inclusion of diffraction mechanism modeling with other propagation mechanisms to do coverage prediction will allow the RF network designers to evaluate the network and enhance coverage, quality, and capacity without the need for costly and extensive drive tests.

Also, it was shown that the diffraction loss and number obstacles are not linearly proportional. The same number of obstacles can result in different diffraction loss due to different arrangement of obstacles. The arrangement of obstacles is parametrized by $h, d_{1}$, and $d_{2}$.

Also, it was seen that the effect of earth curvature has huge impact on diffraction loss if it considered. This is very important fact that should be considered in wireless network design to avoid coverage problems.

It is enough to find the diffraction loss map one time per site with many cells as long as these cells are at the same height. Also, in case of pilot signal power change, antenna tilt, or azimuth change, the diffraction map still valid. Thus the 122 min simulation time will not be required every time.

\section{Conflicts of Interest}

The authors declare that they have no conflicts of interest.

\section{References}

[1] K. Paran and N. Noori, "Tuning of the propagation model ITU-R P.1546 recommendation," Progress in Electromagnetics Research B, vol. 8, pp. 243-255, 2008.

[2] R. K. Crane, Propagation Handbook for Wireless Communication System Design, CRC Press LLC, 2003.

[3] D. A. Bibb, J. Dang, Z. Yun, and M. F. Iskander, "Computational accuracy and speed of some knife-edge diffraction models," in Proceedings of the IEEE Antennas and Propagation Society International Symposium, July 2014.

[4] E. Ostlin, On radio wave propagation measurements and modelling for cellular mobile radio networks [Doctoral Dissertation], Blekinge Institute of Technology, 2009.

[5] M. F. Iskander and Z. Yun, "Propagation prediction models for wireless communication systems," IEEE Transactions on Microwave Theory and Techniques, vol. 50, no. 3, pp. 662-673, 2002.

[6] T. Rappaport, Wireless Communications: Princibles and Practice, Printice Hall, 2nd, 2001.

[7] J. W. McKown and R. L. Hamilton, "Ray tracing as a design tool for radio networks," IEEE Network, vol. 5, no. 6, pp. 27-30, 1991.

[8] T. Schwengler, Wireless \& Cellular Communications, 2010.

[9] K. Bullington, "Radio propagation at frequencies above 30 megacycles," Proceedings of the IRE, vol. 35, no. 10, pp. 1122-1136, 1947.

[10] J. Deygout, "Multiple knife-edge diffraction of microwaves," IEEE Transactions on Antennas and Propagation, vol. 14, no. 4, pp. 480-489, 1966.

[11] J. Epstein and D. W. Peterson, "An experimental study of wave propagation at $850 \mathrm{MC}$," Proceedings of the IRE, vol. 41, no. 5, pp. 595-611, 1953.

[12] J. B. Keller, "Geometrical theory of diffraction," Journal of the Optical Society of America, vol. 52, pp. 116-130, 1962.

[13] W. C. Y. Lee, Mobile Cellular Telecommunications Systems, McGraw-Hill Book Company, 1988.

[14] H. R. Anderson, Fixed Broadband Wireless System Design, John Wiley \& Sons, Chichester, UK, 2003. 


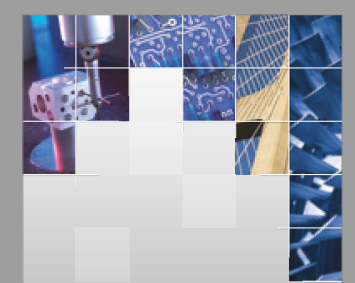

\section{Enfincering}
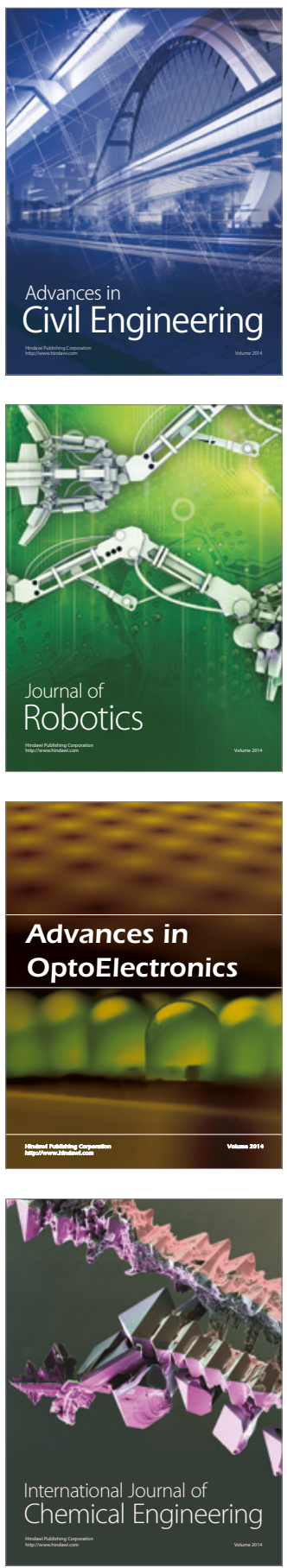

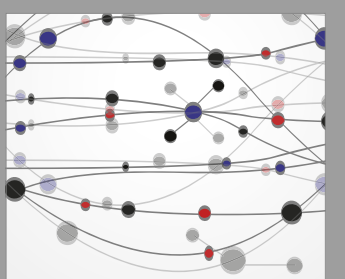

The Scientific World Journal

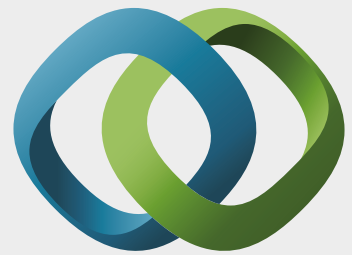

\section{Hindawi}

Submit your manuscripts at

https://www.hindawi.com
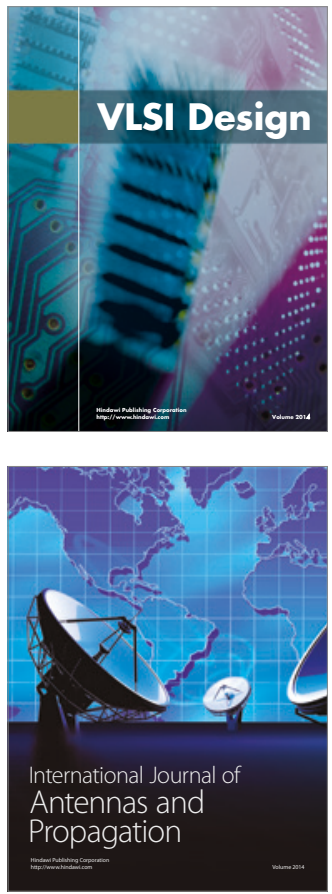

\section{Rotating}

Machinery
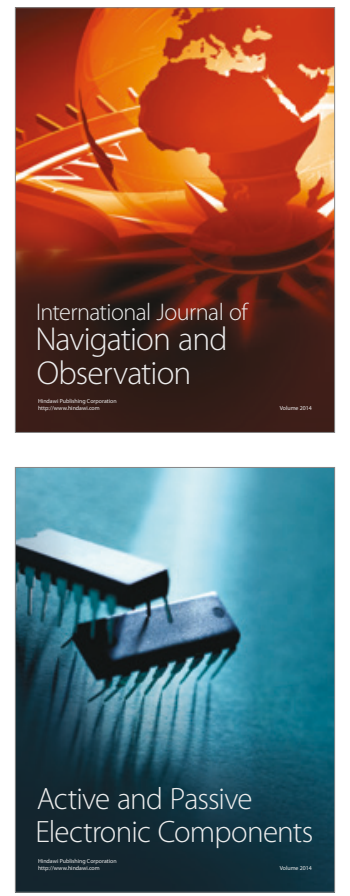
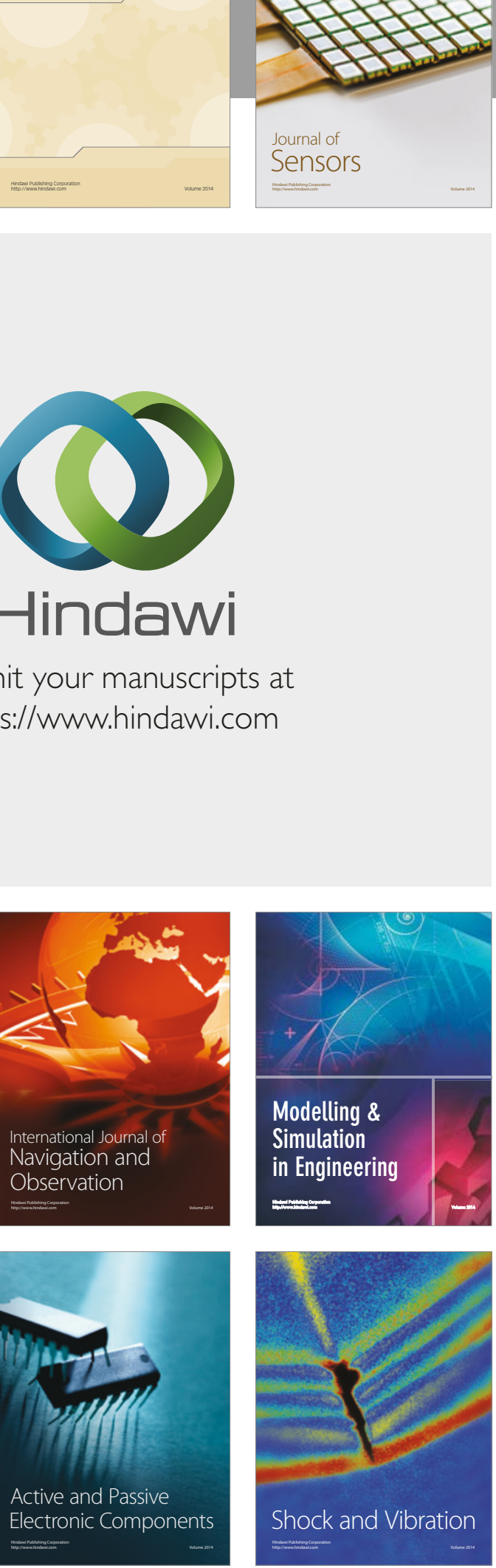
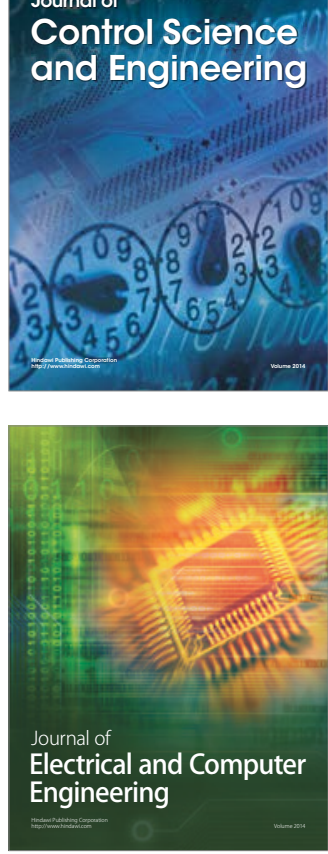

Distributed

Journal of

Control Science

and Engineering
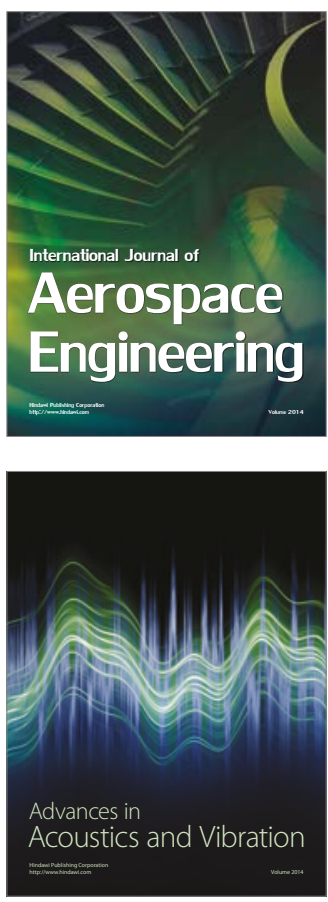

Sensor Networks 